KYUNGPOOK Math. J. 48(2008), 233-240

\title{
On a Background of the Existence of Multi-variable Link Invariants
}

\author{
Fumikazu NAGASATO \\ Department of Mathematics, Meijo University, Shiogamaguchi, Tenpaku, Nagoya \\ 468-8502, Japan \\ e-mail : fukky@ccmfs.meijo-u.ac.jp \\ KANAU HAMAI \\ Kozo Keikaku Engineering Inc. 4-38-13 Hon-cho, Nakano-ku Tokyo 164-0012, \\ Japan \\ e-mail : hamai@kke.co.jp
}

Abstract. We present a quantum theorical background of the existence of multivariable link invariants, for example the Kauffman polynomial, by observing the quantum $(s l(2, \mathbb{C})$, ad)-invariant from the Kontsevich invariant point of view. The background implies that the Kauffman polynomial can be studied by using the $\operatorname{sl}(N, \mathbb{C})$-skein theory similar to the Jones polynomial and the HOMFLY polynomial.

\section{Introduction}

In 1980s-90s, many multi-variable link invariants had been successfully constructed, for example, the $\Lambda$-polynomial ([7]), the $Q$-polynomial ([1], [4]) and the Kauffman polynomial. Why was it possible? In this paper, we present a quantum theorical background of the existence of the above multi-variable link invariants by observing the quantum $(\operatorname{sl}(2, \mathbb{C})$, ad)-invariant from the Kontsevich invariant point of view.

According to [8], the quantum $\left(s o(N), \rho_{0}\right)$-invariant, where $\rho_{0}$ is the fundamental representation of $s o(N)$, is a specialization of the Kauffman polynomial $F(L ; a, z)$ in the Laurent polynomial ring $\mathbb{Z}\left[a, a^{-1}, z, z^{-1}\right]$, which is an invariant of unoriented unframed links defined by the following skein relations with the initial condition $F(\bigcirc ; a, z)=1$ :

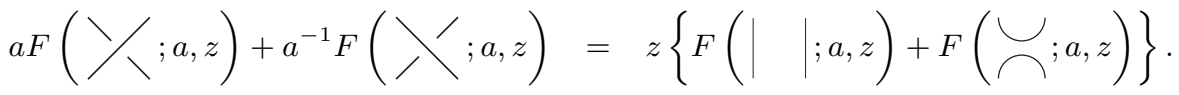

In fact, we can show the equivalence of the weight systems for $\left(s o(3), \rho_{0}\right)$ and $(s l(2, \mathbb{C})$, ad) by using the result in [2], and [8], where ad is the adjoint representation. Then it follows from an analytic ([8]) or a combinatorial observation ([3]) of the Kontsevich invariant that the quantum $\left(s l(2, \mathbb{C})\right.$, ad)-invariant $Q_{s l(2, \mathbb{C}) \text {,ad }}$ is also a specialization of the Kauffman polynomial as well as the the quantum $\left(s o(N), \rho_{0}\right)$-invariant:

Theorem 1.1([3]). The quantum $(s l(2, \mathbb{C})$, ad)-invariant is an unoriented framed link

Received February 21, 2006.

2000 Mathematics Subject Classification: 57M27, 57M25.

Key words and phrases: Kauffman polynomial, $\Lambda$-polynomial, Kontsevich invariant, $Q$-invariant, weight system. 
invariant satisfying the following relations with the initial condition $Q_{s l(2, \mathbb{C}), \text { ad }}(\bigcirc)=e^{h}+$ $e^{-h}+1$ :

$$
\begin{aligned}
Q_{s l(2, \mathbb{C}), \mathrm{ad}}(\backslash)-Q_{s l(2, \mathbb{C}), \mathrm{ad}}(\backslash) & =\left(e^{h}-e^{-h}\right)\left\{Q_{s l(2, \mathbb{C}), \mathrm{ad}}(||)-Q_{s l(2, \mathbb{C}), \mathrm{ad}}(\circlearrowleft)\right\} \\
Q_{s l(2, \mathbb{C}), \mathrm{ad}}(\searrow \bigcirc) & =e^{2 h} Q_{s l(2, \mathbb{C}), \mathrm{ad}}(\mid)
\end{aligned}
$$

Why can $Q_{s l(2, \mathbb{C}) \text {,ad }}$ be thought of as a specialization of the $\Lambda$-, $Q$ - and the Kauffman polynomial? To see this, substitute $z:=e^{h}-e^{-h}, a:=e^{2 h}$ to the relations in Theorem 1.1. Then we get the skein relations of the $\Lambda$-polynomial, except for the sign of the second terms of the both sides of the first relation. The $\Lambda$-polynomial induces the $Q$ - and the Kauffman polynomial. (See [5], for example). In this sense, $Q_{s l(2, \mathbb{C}) \text {,ad }}$ can be regarded as a specialization of the Kauffman polynomial. Moreover, this process explains a quantum theorical background of the existence of the above multi-variable link invariants. The process also implies a possibility that the Kauffman polynomial can be studied by using the $\operatorname{sl}(N, \mathbb{C})$-skein theory similar to the Jones polynomial and the HOMFLY polynomial. (With respect to the $\operatorname{sl}(N, \mathbb{C})$-skein theory, refer to [12]).

In this paper, we concentrate our interest on explaining what we observed in [3]. Namely, we show Theorem 1.1 in a combinatorial way using the Kontsevich invariant, which is different from the method in [8].

\section{Key lemmas}

To prove Theorem 1.1, we use the modified Kontsevich invariant $\widehat{Z}$, the $(\mathfrak{g}, \rho)$-weight system $W_{\mathfrak{g}, \rho}$, its graded version $\widehat{W}_{\mathfrak{g}, \rho}$, quasi-tangles and Jacobi diagrams. There exists an excellent book [11] on these materials, so please refer to the book for details. The following theorem plays an important role in this paper:

Theorem 2.1(Kassel [6], Le and Murakami [9]). The quantum (g, $\rho)$-invariant $Q_{\mathfrak{g}, \rho}$ can be reconstructed by using the composition of the modified Kontsevich invariant $\widehat{Z}$ with the $(\mathfrak{g}, \rho)$-graded weight system $\widehat{W}_{\mathfrak{g}, \rho}$. Namely, $\left.Q_{\mathfrak{g}, \rho}(L)\right|_{q=e^{h}}=\widehat{W}_{\mathfrak{g}, \rho}(\widehat{Z}(L))$ for an arbitrary oriented framed link $L$.

In the final section, we apply this theorem to a proof of Theorem 1.1. Before the application we first focus on the following three key lemmas to Theorem 1.1. For the sake of convenience, we often use the following notation :

$$
H:=\because|\ldots|, \quad P:=\searrow, \quad U:=\nearrow^{\longrightarrow}, \quad 1:=\mid,
$$

where the above diagrams are Jacobi diagrams. We simply denote $W_{s l(2, \mathbb{C}) \text {,ad }}$ by $W$.

Lemma 2.1. The $(s l(2, \mathbb{C})$, ad)-weight system $W$ does not depend on the orientation of support (solid lines) of a Jacobi diagram and is formulated as follows:

(1) $W(\bigcirc)=3$,

(2) $W\left(D \sqcup D^{\prime}\right)=W(D) \cdot W\left(D^{\prime}\right)$, for any Jacobi diagrams $D$ and $D^{\prime}$,

(3) $W(H)=2 W(P)-2 W(U)$. 
Proof. The adjoint representation of $s l(2, \mathbb{C})$ is self-dual, which fact shows that $W$ does not depend on the orientation of support of a Jacobi diagram. (1) and (2) are trivial. (3) is a formula given by Chmutov and Varchenko in [2].

We remark that the first author generalized the formula (3) to a universal $\operatorname{sl}(N, \mathbb{C})$ weight system via the Young symmetrizer in [10].

The formula (3) shows the equivalence of the weight system for $\left(s o(3), \rho_{0}\right)$ and $(\operatorname{sl}(2, \mathbb{C}), \mathrm{ad})$. (Refer to [8]). Although Theorem 1.1 basically follows from the fact, we explain concretely how to prove Theorem 1.1 in a combinatorial way using the Kontsevich invariant.

\section{Lemma 2.2.}

$$
W\left((P-U)^{n}\right)=\frac{1}{2}\left(1-(-1)^{n}\right) W(P)-\frac{1}{3}\left(1-(-2)^{n}\right) W(U)+\frac{1}{2}\left(1+(-1)^{n}\right) W(1)
$$

Proof. This can be immediately shown by induction.

Lemma 2.3. Let $\widehat{W}$ be the $(\operatorname{sl}(2, \mathbb{C})$, ad)-graded weight system. Then there exists a nonconstant element $\lambda=\lambda(h) \in \mathbb{C}[[h]]$ satisfying the following conditions:

$$
\widehat{W} \circ \widehat{Z}(\bigcirc)=3 \lambda, \widehat{W} \circ \widehat{Z}(\bigodot)=\lambda W(U),
$$

where $\bigodot$ is a quasi-tangle with an unspecified orientation and dots with its end points.

Proof. Note that the composition $\widehat{W} \circ \widehat{Z}$ does not depend on the orientation on a quasitangle, which property is derived from Lemma 2.1 , but $\widehat{Z}$ does. Hence, at first we consider an oriented quasi-tangle for $\widehat{Z}$, then ignore the orientation later. In particular, dots with the end points of a quasi-tangle are not essential for $\widehat{W} \circ \widehat{Z}$, so we also ignore them later.

Let us first summarize the definition of the modified Kontsevich invariant $\widehat{Z}$ needed in this proof. For any monomial $w$ in the non-commutative variables $A$ and $B$, the degree of $w$ is defined by its length as a word in $A$ and $B$. Let $\varphi(A, B)$ be the formal power series in the variables $A$ and $B$ as follows:

$$
\begin{aligned}
\varphi(A, B):= & 1+\frac{1}{24}[A, B]-\frac{\zeta(3)}{(2 \pi \sqrt{-1})^{3}}([A,[A, B]+[B,[A, B]]) \\
& +(\text { terms in } A \text { and } B \text { with degree } \geq 4),
\end{aligned}
$$

where $\zeta(z)$ is the zeta function. Let $\nu$ be the Jacobi diagram with support $\downarrow$ as follows:

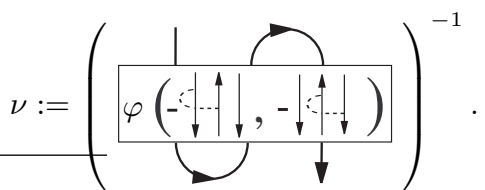

Then the modified Kontsevich invariant $\widehat{Z}\left(\bigcap_{\bullet}\right)$ is defined by

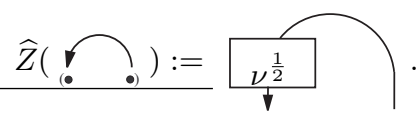


To get a concrete presentation of $\nu$, let us take a closer look at $\nu^{-1}$.

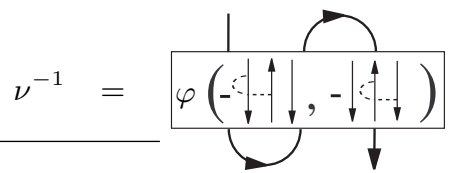

$$
\begin{aligned}
& \left.=1+\frac{1}{24}(\because-1]-1.7\right)+(\text { Jacobi diagrams of degree } \geq 3)
\end{aligned}
$$

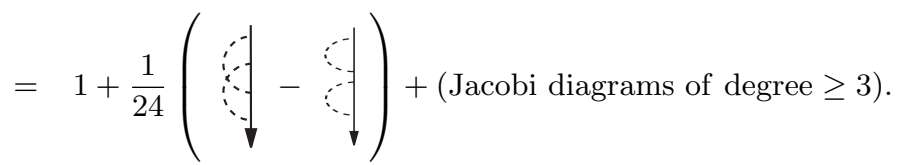

Let us put $\nu:=a_{0}+a_{1}$

For convenience, in the rest of proof, the part (Jacobi diagrams of degree $\geq 3$ ) in the above power series is abbreviated to $R$. Then the following equation holds:

$$
\begin{aligned}
1 & =\nu^{-1} \nu \\
& =\left(1+\frac{1}{24}\left(\begin{array}{r}
\vdots \\
\vdots
\end{array}\right)+R\right)\left(a_{0}+a_{1}\right. \\
& =a_{0}+a_{1} \\
&
\end{aligned}
$$

So we get $a_{0}=1, a_{1}=0, a_{2}=-1 / 24, a_{3}=1 / 24$. Then $\nu$ has the following presentation:

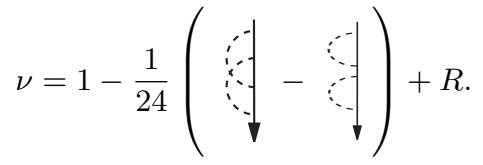

We next focus on the equations below derived from Lemma 2.1,

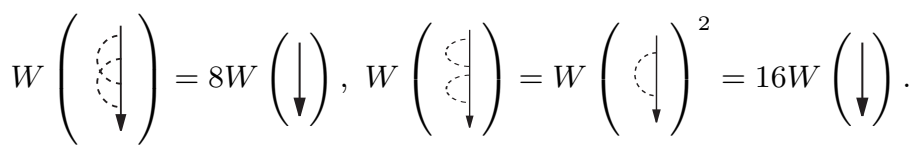

Here we remark that the graded $(s l(2, \mathbb{C})$, ad)-weight system $\widehat{W}(D)$ of a Jacobi diagram $D$ is defined as $h^{\operatorname{deg}(D)} W(D)$, where $\operatorname{deg}(D)$ is a half the number of uni and tri-valent vertices of the graph consisting of all the dashed edges in $D$. Applying these formulas to 
the Jacobi diagram $\nu$, we get

$$
\begin{aligned}
\widehat{W}(\nu) & =\widehat{W}\left(1-\frac{1}{24}\left(\begin{array}{r}
- \\
-
\end{array}\right)+R\right) \\
& =\left(1+\frac{h^{2}}{3}+(\text { terms of degree } \geq 3)\right) W(\downarrow)
\end{aligned}
$$

Note that the second equality of the above equations is derived from Schur's lemma. Let us put $\lambda=\lambda(h):=1+\frac{h^{2}}{3}+($ terms of degree $\geq 3)$. Then we have

$$
\widehat{W} \circ \widehat{Z}(\bigcap)=\widehat{W} \circ \widehat{Z}(\bigcap)=\widehat{W}\left(\frac{\widehat{\nu^{\frac{1}{2}}}}{\nu^{\prime}}\right)=\lambda^{1 / 2} W(\bigcap)
$$

Moreover, we can get the same relation on $\widehat{W} \circ \widehat{Z}(\bigcup)$ as $\widehat{W} \circ \widehat{Z}(\curvearrowleft)$, therefore we finally get the following results:

$$
\begin{aligned}
& \widehat{W} \circ \widehat{Z}(\bigcirc)=\widehat{W} \circ \widehat{Z}(\bigcirc)=\lambda^{1 / 2} W(\curvearrowleft) \circ \lambda^{1 / 2} W(\bigcirc)=\lambda W(\bigcirc)=3 \lambda
\end{aligned}
$$

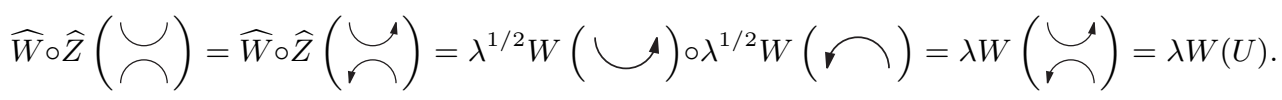

These complete the proof.

\section{Proof of Theorem}

By Theorem 2.1, Lemma 2.1 and the definitions of the modified Kontsevich invariant and the weight system, we see that $Q_{s l(2, \mathbb{C}) \text {,ad }}$ is an unoriented framed link invariant. Moreover, by Theorem 2.1, it suffices to show that

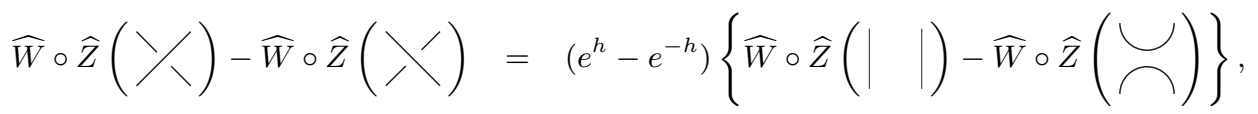

$$
\begin{aligned}
& \widehat{W} \circ \widehat{Z}(\searrow \bigcirc)=e^{2 h} \widehat{W} \circ \widehat{Z}(\mid) \\
& \widehat{W} \circ \widehat{Z}(\bigcirc)=e^{h}+e^{-h}+1,
\end{aligned}
$$

to prove Theorem 1.1. (Refer to the note at the beginning of the proof of Lemma 2.3.) The third equation can be easily checked because

$$
\widehat{W} \circ \widehat{Z}(\bigcirc)=Q_{s l(2, \mathbb{C}), \text { ad }}(\bigcirc)=[3]=\frac{e^{\frac{3 h}{2}}-e^{-\frac{3 h}{2}}}{e^{\frac{h}{2}}-e^{-\frac{h}{2}}}=e^{h}+e^{-h}+1,
$$

where $[n]=\left(e^{\frac{n h}{2}}-e^{-\frac{n h}{2}}\right) /\left(e^{\frac{h}{2}}-e^{-\frac{h}{2}}\right)$ is the quantum dimension. Then Lemma 2.3 shows that $\lambda=\left(e^{h}+e^{-h}+1\right) / 3$. 
Next, let us check the second equation. By the definition of $\widehat{Z}$,

$$
\widehat{Z}(\curlyvee)=\exp \left(\frac{1}{2} \bigcirc\right) .
$$

Recalling the definition of $\widehat{W}$ mentioned in the proof of Lemma 2.3, we obtain the desired equation as follows:

$$
\begin{aligned}
\widehat{W} \circ \widehat{Z}(\searrow \bigcirc) & \left.=\widehat{W} \circ \widehat{Z}\left(\bigcup \bigcup \exp \left(\frac{1}{2}\right)=\widehat{W}\right)\right)=\exp \left(\frac{1}{2} \widehat{W}(\downarrow)\right) \\
& =\exp \left(\frac{4 h}{2} W(\downarrow)\right)=e^{2 h} \widehat{W} \circ \widehat{Z}(\downarrow) .
\end{aligned}
$$

We finally focus on the first equation. By the definitions of $\widehat{W}$ and $\widehat{Z}$,

$$
\begin{aligned}
\widehat{W} \circ \widehat{Z}(\searrow) & =\widehat{W} \circ \widehat{Z}(\searrow)=\widehat{W}\left(P\left(1+\frac{1}{2} H+\frac{1}{8} H^{2}+\cdots+\frac{1}{n ! 2^{n}} H^{n}+\cdots\right)\right) \\
& =W\left(P\left(1+\frac{h}{2} H+\frac{h^{2}}{8} H^{2}+\cdots+\frac{h^{n}}{n ! 2^{n}} H^{n}+\cdots\right)\right) \\
& =W\left(P e^{h H / 2}\right) .
\end{aligned}
$$

By Lemmas 2.1 and 2.2, the following equation holds:

$$
\begin{aligned}
W\left(P e^{h H / 2}\right) & =W\left(P e^{h(P-U)}\right) \\
& =W\left(P \sum \frac{h^{n}}{n !}(P-U)^{n}\right) \\
& =W\left(P \sum \frac{h^{n}}{n !}\left\{\frac{1}{2}\left(1-(-1)^{n}\right) P-\frac{1}{3}\left(1-(-2)^{n}\right) U+\frac{1}{2}\left(e^{h}+e^{-h}\right) \cdot 1\right\}\right) \\
& =W\left(\sum \frac{h^{n}}{n !}\left\{\frac{1}{2}\left(1-(-1)^{n}\right) \cdot 1-\frac{1}{3}\left(1-(-2)^{n}\right) U+\frac{1}{2}\left(1+(-1)^{n}\right) P\right\}\right) \\
& =\frac{1}{2}\left(e^{h}-e^{-h}\right) W(1)-\frac{1}{3}\left(e^{h}-e^{-2 h}\right) W(U)+\frac{1}{2}\left(e^{h}+e^{-h}\right) W(P) .
\end{aligned}
$$

Similarly, we can get the following relation:

$$
\begin{aligned}
\widehat{W} \circ \widehat{Z}(\searrow \prime) & =\widehat{W} \circ \widehat{Z}(\searrow) \\
& =\frac{1}{2}\left(e^{-h}-e^{h}\right) W(1)-\frac{1}{3}\left(e^{-h}-e^{2 h}\right) W(U)+\frac{1}{2}\left(e^{-h}+e^{h}\right) W(P) .
\end{aligned}
$$


Hence, by Lemma 2.3, we obtain the following equation:

$$
\begin{aligned}
& \widehat{W} \circ \widehat{Z}(\backslash \backslash)-\widehat{W} \circ \widehat{Z}(\backslash \backslash) \\
= & \left(e^{h}-e^{-h}\right) W(1)-\frac{1}{3}\left(e^{2 h}-e^{-2 h}+e^{h}-e^{-h}\right) W(U) \\
= & \left(e^{h}-e^{-h}\right)\left\{W(1)-\frac{1}{3}\left(e^{h}+e^{-h}+1\right) W(U)\right\} \\
= & \left(e^{h}-e^{-h}\right)\left\{\widehat{W} \circ \widehat{Z}(||)-\frac{1}{3 \lambda}\left(e^{h}+e^{-h}+1\right) \widehat{W} \circ \widehat{Z}\left(\bigcirc^{\bigcirc}\right)\right\} .
\end{aligned}
$$

Recall that $\lambda=\left(e^{h}+e^{-h}+1\right) / 3$. Therefore this equation completes the proof of the first equation and Theorem 1.1.

Acknowledgment. We would like to thank Professor Mitsuyoshi Kato for his encouragements. The first author has been supported by JSPS Research Fellowships for Young Scientists.

\section{References}

[1] R. D. Brandt, W. B. R. Lickorich and K. C. Millett, A polynomial invariant for unoriented knots and links, Invent. Math., 84(1986), 563-574.

[2] S. V. Chmutov and A. N. Varchenko, Remarks on the Vassiliev knot invariants coming from $\mathrm{sl}_{2}$, Topology, 36(1997), 153-178.

[3] K. Hamai, A combinatorial reconstruction of the quantum $(\operatorname{sl}(2, \mathbb{C}), a d)$-invariant through the Kontsevich invariant (in Japanese, joint work with F. Nagasato), Master thesis, Kyushu University (2002).

[4] C. F. Ho, A new polynomial for knots and links-preliminary report, Abstracts Amer. Math. Soc., 6(1985), 300.

[5] T. Kanenobu, Tangle surgeries on the double of a tangle and link polynomials, Kobe J. Math., 19(2002), 1-19.

[6] C. Kassel, Quantum Groups, GTM 155, Springer-Verlag, 1995.

[7] L. H. Kauffman, On knots, Ann. of Math. Studies, 115, Princeton University Press, 1987.

[8] T. T. Q. Le and J. Murakami, Kontsevich's integral for the Kauffman polynomial, Nagoya Math. J., 142(1996), 39-65.

[9] T. T. Q. Le and J. Murakami, The universal Vassiliev-Kontsevich invariant for framed oriented links, Compositio Mathematica, 102(1996), 41-64.

[10] F. Nagasato, A diagrammatic construction of the $(\operatorname{sl}(N, \mathbb{C}), \rho)$-weight system, Interdisciplinary Information Sciences, 9(2003), 43-51. 
[11] T. Ohtsuki, Quantum Invariants, Series on Knots and Everything, 29, World Scientific, 2002.

[12] Y. Yokota, Skeins and quantum SU(N) invariants of 3-manifolds, Math. Ann., 307(1997), 109-138. 